\title{
First-time demonstration of measuring concrete prestress levels with metal packaged fibre optic sensors
}

\author{
I. Mckeeman ${ }^{1}$, G. Fusiek ${ }^{1}$, M. Perry ${ }^{2}$, M. Johnston ${ }^{3}$, M.

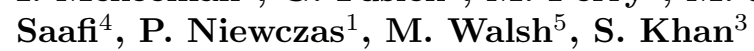 \\ ${ }^{1}$ Department of Electronic and Electrical Engineering, Technology and \\ Innovation Building, University of Strathclyde, Glasgow, G1 1RD, UK \\ 2 Department of Civil and Environmental Engineering, James Weir Building, \\ University of Strathclyde, Glasgow, G1 1XJ, UK \\ 4 Department of Engineering, Lancaster University, Lancaster LA1 4YR, UK \\ 3 Civil Design Group, EDF Energy Nuclear Generation Ltd, East Kilbride, UK \\ 5 Vinci Technology Centre UK Ltd, Leighton Buzzard, UK \\ E-mail: iain.mckeeman@strath.ac.uk
}

\begin{abstract}
.
In this work we present the first large-scale demonstration of metal packaged fibre Bragg grating sensors developed to monitor prestress levels in prestressed concrete. To validate the technology, strain and temperature sensors were mounted on steel prestressing strands in concrete beams and stressed up to $60 \%$ of the ultimate tensile strength of the strand. We discuss the methods and calibration procedures used to fabricate and attach the temperature and strain sensors. The use of induction brazing for packaging the fibre Bragg gratings and welding the sensors to prestressing strands eliminates the use of epoxy, making the technique suitable for high-stress monitoring in an irradiated, harsh industrial environment. Initial results based on the first week of data after stressing the beams show the strain sensors are able to monitor prestress levels in ambient conditions.
\end{abstract}




\section{Introduction}

Prestressed concrete pressure vessels (PCPVs) and prestressed concrete containments (PCCs) on a nuclear power plant serve safety-critical functions, shielding the outside environment from radiation and containing high pressures and temperatures during both normal operation and any fault conditions. Prestressed tendons consisting of strands, which are made up of high tensile steel wires, are used to prestress these structures to the appropriate level. The strands provide the compressive force required to compensate for concrete's inherent tensile weakness. As a consequence, monitoring strand prestress levels is an essential part of a PCPV's or a PCC's maintenance schedule [1].

In existing nuclear containments in the UK, prestressing strand ducts are ungrouted, and so a small percentage (1-2\%) of the unbonded strands can undergo direct force measurements using a prestressing jack. This labour-intensive practice is termed the lift off technique. Globally, however, it is more common for containment ducts to be grouted with cement. In these cases, load cells are instead installed onto several ungrouted strands [2]. In some cases, vibrating wire strain gauges, installed within the containments during manufacture, are used to verify prestress measurements. However these instrumentation systems are used tentatively as they wear out or decalibrate over time. All of these existing practices are not ideal due to their high cost and assumption that a small percentage of monitored strands is representative of the population. Furthermore, all of these techniques provide a measurement of the averaged prestress over the entire strand. But it is well-known that prestress can show large variations over a strand's length due to the effects of strand-duct and inter-strand friction, see Section 2. There is therefore an urgent need to develop an economical, reliable and retrofittable prestress monitoring system which can survive a warm and radioactive environment and provide spatially distributed force measurements along a large population of strands.

Recently, optical sensors and particularly fibre Bragg gratings (FBGs) have been gaining in popularity to provide distributed measurement of strain and temperature in harsh conditions [3]. Within a nuclear and electricity generation context, optical fibre sensors may provide a unique solution to sensing problems due to their intrinsic properties such as excellent multiplexing potential, immunity to electromagnetic interference, safe mode of operation and radiation resistance [4]. Fibre Bragg gratings are well suited to strain sensing applications especially in structural health monitoring (SHM) [5, 6].

There are several different techniques for monitoring prestressing tendons with FBGs. Prestress loss can be estimated by placing strain sensors into the concrete surrounding the tendons [7], where stress levels are lower, but the technique requires several generalized assumptions and does not allow for retrofitting. Alternatively, the FBGs can be mounted onto the tendons giving a direct localized strain measurement. This reduces the assumptions and consequently increases the accuracy of the measurement. However, this subjects the sensors to greater strains and so correct packaging is essential if they are to survive installation and remain reliable for years afterwards. Several studies have shown that it is possible to replace the central wire in a steel prestressing strand with one made from a fibre reinforced polymer (FRP) wire with an FBG embedded into it $[8,9,10]$. This may protect the sensors from damage, but replacing the central wire changes tensile and load bearing properties of the strands. FRPs may also be unsuitable for nuclear applications due to their low shear strength and unproven radiation hardness [11, 12]. Retrofitting sensors to standard, steel prestressing strands is another potentially cheaper universally available option, but one that is rarely investigated due to a lack of suitable attachment techniques.

Here we demonstrate all-metal optical sensor packaging and attachment to prestressing strands; this method removes the need for epoxy bonding in an etched groove, an example stated as inferior to embedded sensors [10]. The calibration procedure needed for the sensors to monitor the concrete beam for an extended period of time under ambient conditions is also described. This is the first time these sensors have been fully multiplexed, attached to seven wire strands and stressed up to $60 \%$ of the ultimate tensile strength (UTS) of the strands $(230 \mathrm{kN})$ inside a prestressed concrete structure. 
First-time demonstration of measuring concrete prestress levels with metal packaged distributed fibre optic sensors3

\section{Concrete set up}

\subsection{Concrete beam design}

To assess the performance of the metal packaged strain and temperature sensors in an environment similar to the intended application, two 2.4 metre prestressed concrete beams were cast for the sensors to be tested on. Each beam had two ducts running through it where prestressing strands were inserted. One beam contained straight ducts, the other had a double harp duct geometry, Fig.1. The bent ducts allowed the effects of duct friction to be investigated (see section 2.2.2). Several rebars ran through the beams with rebar cages at each end to provide strength for the concrete beams whilst being moved and to guard against failure upon stressing. The prestressing strands were $18 \mathrm{~mm}$ diameter cold drawn 7 wire strands, manufactured according to the BS 5896:1980 standard. After the beams were stressed they exhibited prestress loss effects similar to those in a prestressed containment.

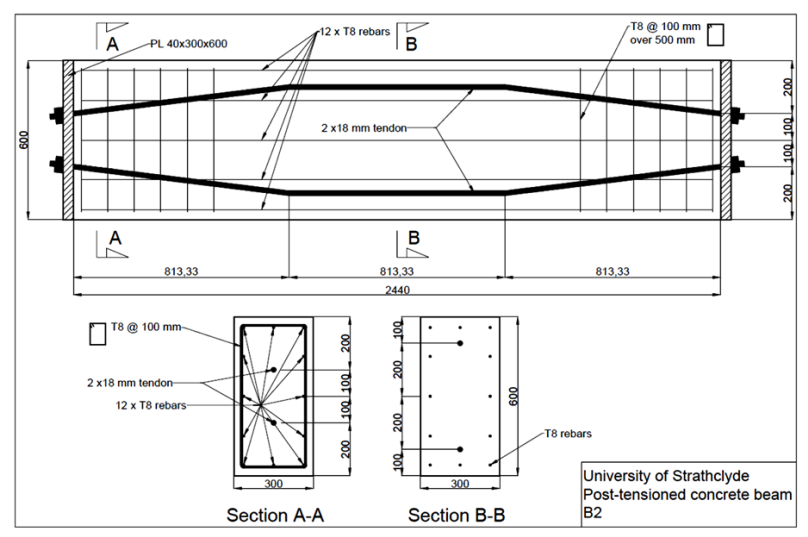

Figure 1. The harped-duct concrete beam dimensions. The straight duct concrete beam had exactly the same dimensions but with straight ducts spaced $300 \mathrm{~mm}$ apart.

\subsection{Prestress loss in concrete}

The tensile strength of a concrete structures is often increased using prestressed tendons in safety critical structures, such as bridges and nuclear containment buildings. The steel tendons or cables are slotted through ducts cast into the structure and then tensioned, terminating at anchorages which compress the concrete [13]. Tendon ducts are then either grouted with the aim of transferring more stress to the concrete, or filled with oil to prevent strand corrosion allowing load measurement and potential replacement of the tendons. Due to the forces involved, the structure adjusts to the compressional stress and the result is seen as a combination of several different definable effects. Over time these effects contribute to the decrease in prestressing force of the steel tendons. The effects can be grouped into two categories: instantaneous and time dependent. Instantaneous effects occur at the time of stressing and include elastic effects such as concrete compression and also friction on the tendon and anchor slippage. Time dependent effects occur over many years and are affected by environmental factors such as temperature and humidity. These effects include concrete creep and shrinkage and steel relaxation. It is hard to predict the exact prestress loss in a structure at any given time because of variation in the environmental conditions and anisotropy in the structure. Therefore, it is necessary to monitor prestressed concrete structures and traditionally this work is carried out through manual inspection.

2.2.1. Anchor Slippage During the stressing procedure, the prestressing tendons undergo an unavoidable loss of extension when they are locked off at the anchorage points. The magnitude of the anchor slippage is dependent on the length of the prestressing strand.

$F_{a s}=\frac{\Delta_{A}}{L} E_{P S}$

Here $\Delta_{A}$ is the magnitude of the anchor slip, $L$ is the length of the tendon and $E_{P S}$ is the Youngs modulus of steel. From equation 1 it is clear there is a larger prestress loss for short lengths of strand [13].

2.2.2. Friction Friction in the tendon ducts reduces the prestress level as a function of distance from the stressed end. The effects of friction cannot be measured by existing methods and are generally assumed from standard values $[14,15,16]$. Friction can be caused by curvature of a duct and also by unintentional wobbles in the duct trajectory resulting from imperfect construction. These two effects are captured in Cooley's formula [17]:

$F_{f}(l)=F_{i}\left(1-e x p^{-\mu \alpha-K l}\right)$

$F_{f}(l)$ is the force at distance $l$ from the jack end. $F_{i}$ is the force in the strand at the jack end, $\mu$ is the coefficient of friction between the tendon and duct, $\alpha$ is the angle that the strand has turned through. While $K$ is the wobble factor of the duct. Using equation 2 the force at different points along a tendon can be calculated assuming the duct geometry is known. Fig. 2 is a graph of the force distribution predicted for the bent ducts in this experiment. The coefficients are assumed to be $\mu=0.2$ and $K=6.5 e-4$, which are typical values for the strands and ducts. 


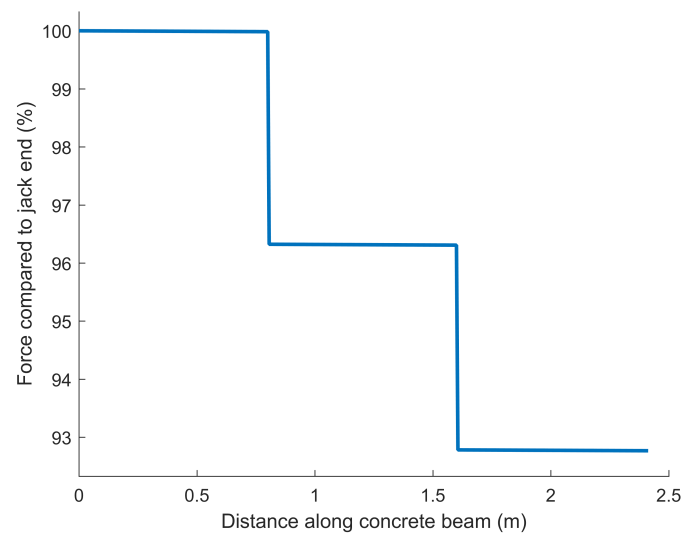

Figure 2. Prediction of prestress loss due to friction in a double harped tendon duct.

\section{Sensing architecture}

In this work, FBG sensors were used to monitor distributed prestress loss in strands as they were stressed in the mock-up beam.

\subsection{Strain and temperature sensing with FBGs}

An FBG is a periodic variation in refractive index written into an optical fibre. The varying refractive index reflects a specific wavelength of light depending on the spacing and the difference in refractive index of the grating. As a result the peak wavelength responds to changes in the periodicity of the grating caused by temperature or strain.

$\frac{\Delta \lambda_{B}}{\lambda_{B}}=K_{\varepsilon} \Delta \varepsilon+K_{T} \Delta T$

Where $K_{\varepsilon}(0.78)$ and $K_{T}\left(6.7 \mathrm{ppm} /{ }^{\circ} \mathrm{C}\right)$ are the strain and temperature sensitivity of an FBG [18]. Therefore, temperature shifts measured by a temperature sensor can be used to determine the strain, $\Delta \varepsilon$ independent of ambient temperature fluctuations. FBGs are discrete sensors, but can be easily multiplexed allowing a quasi-distributed measurement and are widely used to measure strain. It must be noted that, for applications in civil engineering, correct packaging is very important if the FBGs are to remain functional in the field [19]. This is because silica glass is brittle and so is degraded by surface damage [20].

\subsection{Sensor design and fabrication}

The sensor fabrication process for this test has been previously documented [21, 22]. Fibre Bragg gratings, written in metal coated fibre, were brazed into $25 \mathrm{~mm}$ metal capillaries using an induction coil in order to mechanically and chemically protect them from the environment. Additionally, by using a metal packaging the radiation resistance is increased compared to polymer encapsulation [4]. Steel heat susceptors were placed at the end of the capillaries to concentrate heat around the brazing joint to melt the brazing solder. This process was optimised to obtain the required capillary action of the brazing material into the metal capillaries without reducing the structural integrity of the optical fibre due to heat exposure. The capillary encapsulated FBGs were used as temperature sensors, see Fig.3; they were attached to the steel strands using silicone sealant at one end to ensure strain was not transferred to the FBG. The strain sensors had metal shims (10mm wide) brazed onto each end of the capillary. The shims were then spot welded onto the strands, see Fig.3. It should be noted that previous studies revealed that spot welding the sensors to the strands does not negatively impact on the strand strength [23].

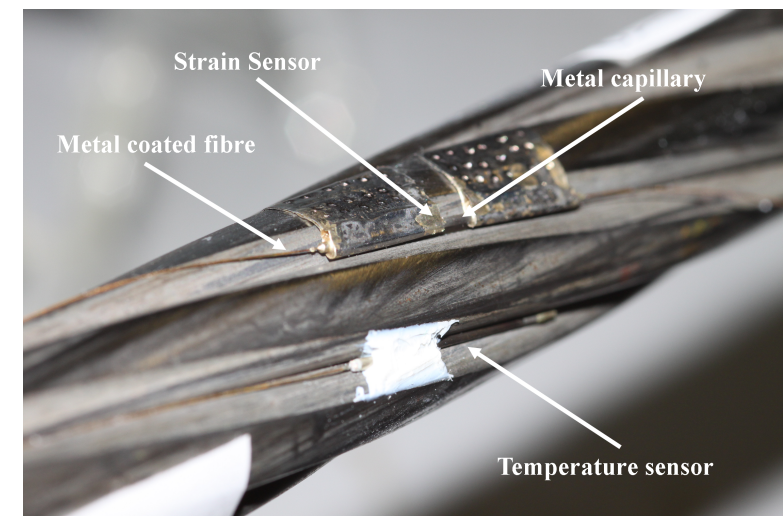

Figure 3. Strain sensor and temperature sensor attached to a prestressing strand.

To reduce the creep rate of the sensor joints when subject to high stress levels (the steel strands are routinely stressed to $80 \%$ UTS of steel), a high temperature brazing solder was utilised $[24,25]$. This ensures the sensors experience smaller creep under prolonged loading [26], especially compared to epoxy bonds stressed above 50\% UTS [27]. The melting point of the solder is around $650^{\circ} \mathrm{C}$. Therefore, the fibre exposure time required careful control as conventional FBGs are thermally erased at high temperatures [28]. A sample of four sensors was monitored for reflectivity losses during the fabrication process (one can be seen in Fig.4).

The wavelength response of the FBG during capillary encapsulation shows the temperature was capped at a certain value due to the Curie transition in kovar [21] as seen in Fig.4 (peaks 1 \& 2 in the bottom graph). When the shims were attached to the capillaries, the susceptors were not used as the shims themselves act as heat concentrators. The observed wavelength response is not capped (see Fig.4 bottom 
graph peaks $3 \& 4$ ) due to the thermal expansion of the capillary straining the FBG. Furthermore, the shims made a physical contact with the capillary, whereby heat was transmitted more readily to the FBG. On average the peak reflection reduction of the FBGs was $35 \%$, Fig.4, showing good agreement with previous studies [21] and further confirming the suitability of this technique to protect FBGs for temperature and strain sensing. The capillary-shim joint was tested to failure with a stressing vice three times. The metal capillary failed first, proving the brazing process can create shim-capillary joints stronger than the tensile strength of the metal capillaries.

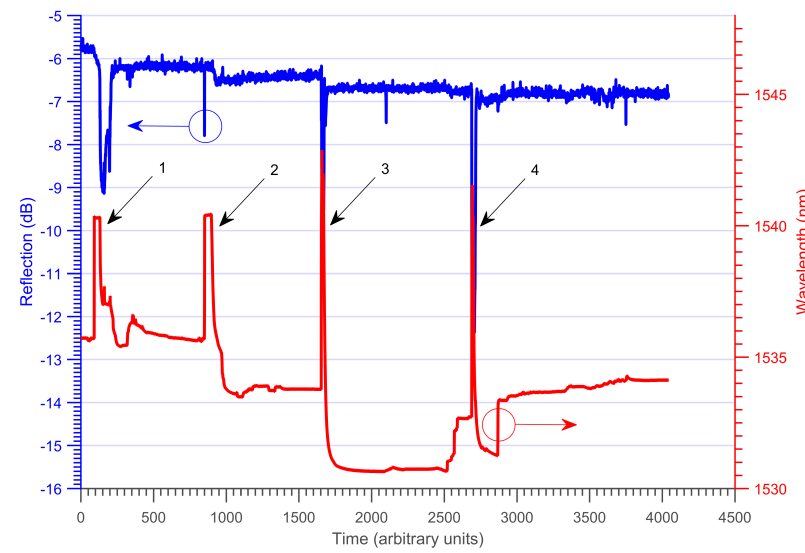

Figure 4. Wavelength and reflection response during strain sensor packaging. Peaks 1 and 2 are due to the FBG being encapsulated in the capillary. Peaks 3 and 4 result from shim attachment.

\subsection{Fibre layout on the prestressing strands}

Each pre-stressing strand had three strain and three temperature sensors equidistantly attached to it, Fig.5. The strain and temperature sensors were connected in series with each other to form two fibre lines. The fibre connecting the sensors was helically wound round the strands and sat within the strand grooves ensuring it was protected. The two fibre lines, one strain and one temperature, were connected outside the beam for troubleshooting of the sensor lines. This allowed any potential breakages of either fibres or sensors to be pin pointed.

Each instrumented strand was interrogated on one optical channel of the interrogator, meaning six FBG peaks were detected on each channel. Additionally load cells at either end of the beam measured the force in the strands at the bearing plate. It is important to measure the force (and its loss) at the end of the strand because relaxation of steel by definition is a loss of stress at constant strain. Therefore the strain sensors are not able to measure the steel relaxation component of the

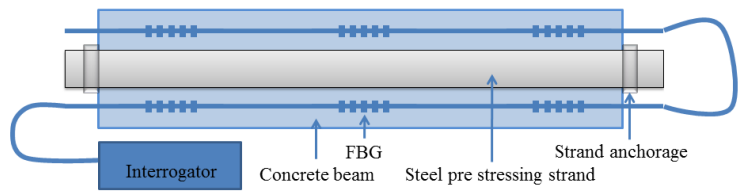

Figure 5. Sensor layout on the strands, the temperature and strain sensors are on separate fibre lines.

prestress loss. Complimentary load cell force and strain sensor strain measurements are able to determine the complete prestress loss while providing a distributed measurement of stress throughout the strand.

\subsection{Thermal calibration}

Temperature calibration of the temperature and strain sensors was required to compensate for spurious thermal effects in equation 3 . The temperature sensors were calibrated before being attached to the strands, whilst the strain sensors were calibrated insitu by induction heating. A J-type thermocouple was used as the temperature reference for all the sensors; (resolution $0.1^{\circ} \mathrm{C}$ ). To achieve a better accuracy and lower error from the FBGs a more accurate temperature reference such as a platinum resistance thermometer could be used (accuracies of $\pm 0.02^{\circ} \mathrm{C}$ are readily achievable).

3.4.1. Calibration of temperature sensors The twelve temperature FBG sensors for the concrete beam test were calibrated at the same time in an environmental chamber with an accuracy better than $1^{\circ} \mathrm{C}$. They were subject to four heating cycles from $10-50^{\circ} \mathrm{C}$. As the thermocouple and FBGs have very different thermal conductivities, both were mounted on a single aluminium plate to ensure even heating at the same rate during calibration. The temperature was stepped in $10^{\circ} \mathrm{C}$ intervals and held at each point for two hours to allow the temperature to stabilise. An average temperature sensitivity of $15.2 \mathrm{pm} /{ }^{\circ} \mathrm{C}$ was determined. Sensors with defective brazing joints can be discovered during the temperature calibration process [29].

3.4.2. Calibration of strain sensors Generally the structures monitored by strain sensors are large; therefore, controlled temperature characterisation of individual strain sensors is challenging. For this test the stressing strands were $4 \mathrm{~m}$ long. In field applications, sensors are often assumed to have the same temperature response. However, verifying a sensor's response allows the measurement accuracy of 
First-time demonstration of measuring concrete prestress levels with metal packaged distributed fibre optic sensors6

individual sensors to be improved. To calibrate the strain sensors for this experiment a portable, in-situ method for thermally calibrating FBG sensors which are attached to pre-stressing strands was developed and applied [30].

\subsection{Strain sensor relaxation}

It is likely that the brazing process introduces some residual thermal stress in the brazed joints [31]. This is due to the high temperature needed to melt the brazing solder and the subsequent rapid cooling of the joint. In our previous work an instrumented strand underwent four stress cycles up to $1300 \mathrm{MPa}$. The strand was held at $1300 \mathrm{MPa}$ for four minutes each cycle. It was found that the sensor response exhibited relaxation characteristics which were attributed to the relaxation of residual stress within the brazed joints [23].

As a consequence, the strain sensors for this test underwent a series of stressing cycles on a custom made vice, Fig.6. This helped to relax the brazed joints before being attached to the stressing strands, allowing the sensors to measure strain with minimised drifts in measured strain. The vice was designed so that the contact surface where the shims were attached matched the geometry of the tendon surface Fig.6a. The sensors were mounted onto the vice and stressed so that the peak wavelength shifted by about $3.5 \mathrm{~nm}$ (corresponding to a strain of $4.5 \mathrm{~m} \varepsilon$ assuming the strain coefficient was that of bare optical fibre, $K_{\varepsilon}=0.78$ ). They were held for five minutes and this was repeated until long term drifts were negligible.

Whilst stressing the sensors on the vice, relaxation was clearly seen (first stress cycle of Fig.7). This was characterised by a decrease in strain similar to that seen in steel. Another important feature revealed through these tests was that once the sensors had been stressed, if the subsequent stressing was below the maximum wavelength reached previously the relaxation was reduced. This can be clearly seen in Fig.7 where stressing cycle two and four reach a smaller maximum wavelength than the previous stressing, indicating that the stressing vice is able to relax the sensors to a significant extent. This suggests the sensors should be stressed above the level of stress they will experience during service, allowing the relaxation in service to be significantly reduced to measure tendon strain more accurately from the beginning.

\subsection{Interrogator and data recording}

The FBG sensors were interrogated with a commercial interrogator from National Instruments (PXIe-4844) housed within a PXI chassis. The interrogator had four channels with a $10 \mathrm{~Hz}$ sampling rate; it
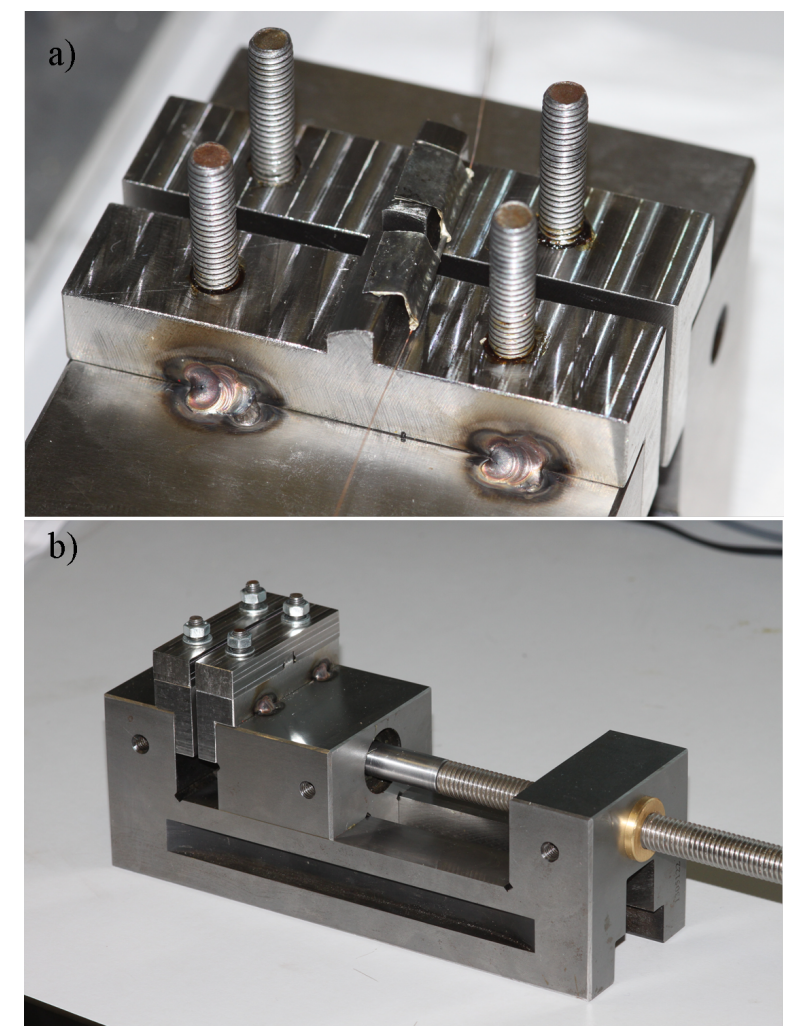

Figure 6. The stressing vice used to relax the strain sensors, with surface geometry matching that of the strand wires.

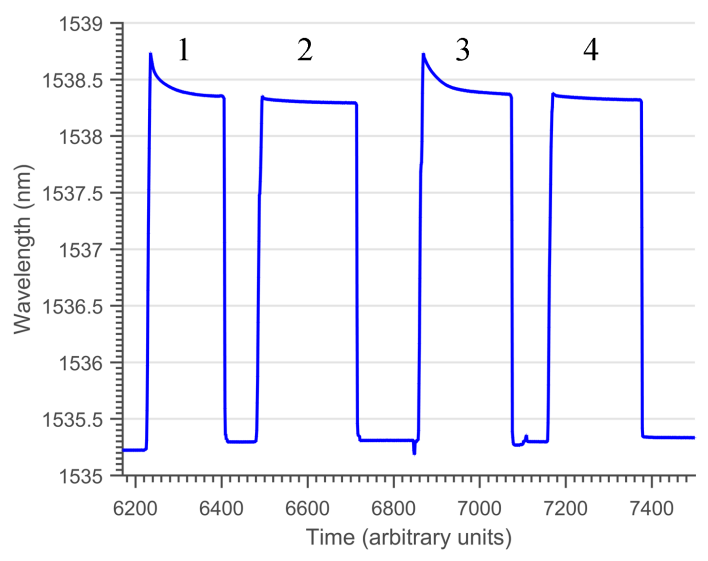

Figure 7. Demonstration of sensor relaxation. Reduced sensor relaxation is seen on peaks 2 and 4 because the sensor was subject to a higher strain previously (peak 1 and 3 ).

had a peak detection accuracy and repeatability of $1 \mathrm{pm}$ (equivalent to a strain of $\sim 1 \mu \varepsilon$ ). The load cells were measured from the same PXI chassis allowing high levels of synchronisation between the optical and electrical measurements by utilizing a $10 \mathrm{MHz}$ on board reference clock. The PXI unit was connected to a desktop computer via an Ethernet cable allowing remote monitoring of the concrete beams. It is envisaged that a commercial system 
First-time demonstration of measuring concrete prestress levels with metal packaged distributed fibre optic sensors7

would require some form of automatic gain to equalise the reflectivities or adjust the detection threshold for each FBG individually. Such techniques are readily available.

\section{Strand installation and stressing procedure}

\subsection{Strand installation}

On a nuclear power site strand installation follows a strict procedure. This was replicated as closely as possible during the test. Therefore the instrumented strands were inserted into the tendon ducts with interrogating fibres attached. Optical fibres exiting the ends of the strands were fed through the ducts first. A robust method for attaching the interrogating optical cable was demonstrated. It utilised high strength optical fibre cable whilst being low profile to allow the stress jack to slide over it.

The stress jack was calibrated daily by a traceable standard hollow load cell; this ensured the stress jack measured force correctly. Wedges were seated in the barrel at the dead end of the strand, the stress jack was then inserted onto the live end of the tendon. First, the reinforced optical fibre cable was threaded through the stress jack. The optical fibre pigtails were reconnected and the sensors were interrogated for the stressing of the strands.

\subsection{Strain calibration of strain sensors}

Before the final stressing, the strands underwent one load cycle to calibrate the strain sensors. Calibration of the sensors when they are permanently attached to the host object is desirable so the strain transfer from the strand to the sensor can be determined. The strain transfer is dependent on the sensor package geometry, the attachment method and the interaction of shear strain between the components [32, 33]. Small fabrication and attachment differences between sensors mean that a universal strain transfer ratio would not be accurate. (Note that if an automated sensor fabrication facility was developed, capable of manufacturing devices with highly consistent behavior, on-site calibration could possibly be avoided.) Therefore, calibration on site is necessary to achieve the greatest performance. For the calibration cycle, the load was increased in $20 \mathrm{kN}$ steps and held at each point for a few 10 s of seconds. Once the maximum load had been reached the stress jack reduced the load down to zero in $40 \mathrm{kN}$ steps. The FBG sensors and load cells were compared to the force measured on the stress jack.

The final stressing procedure followed the calibration cycle; however, barrels and wedges were inserted onto the live end. The strand was tensioned in $20 \mathrm{kN}$ intervals up to the desired force when the wedges were seated in the barrel and the strand was locked off. The FBGs and load cells were monitored throughout the process allowing the calibration cycles to be checked against the final stressing.

\section{Results}

\subsection{Strain sensor calibration}

A typical example of the sensor response for a strain sensor plotted against the load cell at the end of the strand is shown in Fig.8. The sensor response shows two distinct stages at low force and high force. At low force (up to $\sim 125 \mathrm{kN}$ ) the strain shows a linear relationship to force measured by the load cell. At higher force the response is also relatively linear however the slope coefficient is larger (corresponding to $K_{\varepsilon}=0.56$ ). This response was consistent for all the sensors monitored. For each sensor the point at which the slope of the line of best fit changed was different; however, it was always in the $100-150 \mathrm{kN}$ range. Whilst testing with a single metal rod this characteristic was not seen and the strain sensor response was linear up to stresses of $1300 \mathrm{MPa}$ [23, 22] (equivalent to approximately $300 \mathrm{kN}$ in the pre stressing strands). This shows that possibly the strain sensors cannot be reliably calibrated by the total force measured at the end of the 7 -wire strand. This is discussed in more detail in section 6.1 .

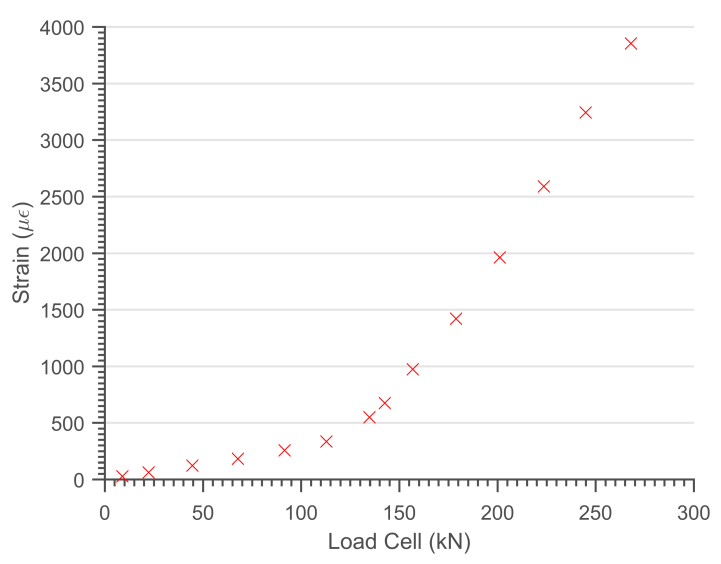

Figure 8. Strain of an individual wire measured by a strain sensor compared to the force of the 7 -wire strand measured by the load cell during the calibration cycle.

\subsection{Anchor slippage}

After the stress jack tensioned the strand to the required force, the wedges at the live end were seated hydraulically by the jack. The resulting anchor slippage was clearly seen on the load cells and strain 


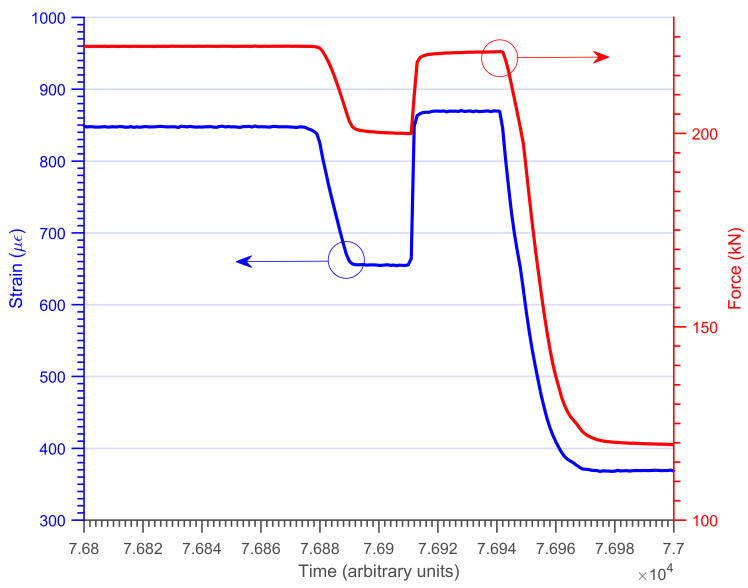

Figure 9. Anchor slippage measured by a load cell (top) and a strain sensor (bottom).

sensors. Figure 9 shows an example of this; here the magnitude of the force/strain dip before the wedges were seated in the barrel varies slightly between the strain sensor and load cell. This is possibly due to non-uniform force distribution between the wires of the strand. The wedge draw in was large due to the short length of the concrete beams.

\subsection{Time dependent prestress loss}

The temperature during the experiment was recorded by the temperature FBG sensors and by a thermocouple that was mounted to the outside of one of the concrete beams, Fig.10. The temperature FBG sensors correspond well with the thermocouple but lag behind it slightly because the room temperature fluctuations take time to affect the temperature inside the strand ducts in the concrete beam. The load cell force can be seen in Fig.11, this is the total force in the strand. The strain sensors were compensated with their corresponding temperature sensors using equation 3 . The result of the compensation is shown in Fig.11. It relates well with the load cell force as both measure a decrease in prestress. The load cell should measure a larger force decrease because steel relaxation also contributes to the prestress loss.

Temperature fluctuations can be seen in both the load cell force and the strain sensor. The load cell was not compensated for temperature fluctuations so this is due to the intrinsic temperature sensitivity of the load cell and the thermal expansion of the concrete beam and strands. The strain sensor has been compensated for temperature but there is still some residual influence. This is because the temperature compensation coefficient does not fully account for all the temperature dependencies of the concrete beam. The temperature coefficient was obtained whilst the strain sensors were attached to the steel strands, so it accounts for the intrinsic temperature sensitivities of the sensor and also the thermal expansion of the prestressing strand. Therefore the fluctuations seen are due to concrete and anchor plate thermal expansion.

\section{Discussion}

\subsection{Strain sensor calibration}

Calibration of the strain sensors is an important aspect of sensor development. Currently an independent calibration method has not been developed due to the difficulty of this task. Strain calibration of the sensors outside of the vessel is vital for identifying the experimental load profile of the strand without assuming that Cooley's formula holds true. One of the main advantages of distributed strain measurement is that Cooley's formula and the assumptions of friction coefficients can be tested; therefore, a robust calibration method should be investigated. Ideally, the section of strand where the sensor is attached would be tensioned in a tensile machine with an external measurement of strain allowing a direct correlation of sensor wavelength to strain in the strand. However this is not a practical solution due to the size of the strands and potential damage on the strands by the tensile machine grips.

For this experiment the strain sensors were calibrated from the load cell measurements as the force in the tendon was converted into a strain. For the straight ducts it was assumed that there was a constant strain throughout the tendon. However for the bent ducts friction reduces the load on the strand at the dead end compared to the live end as seen in the theory section. As the geometry of the duct is known, as well as the force at the live end due to the stress jack, the distribution of the load in the strand could be estimated. This was checked against the readings of the load cells at each end of the strand; the calculations predict that there should be a $5 \%$ decrease in force at the dead end. However, the force difference between the load cells was larger than this (8-15\% difference). Additionally, in general, the strain measured by the sensors was lower than that expected from the calculations. This could be due to the strain transfer of the sensors which is affected by the helix angle of the outer wires.

\subsection{Sensor fibre line breakages}

During installation and stressing of the strands a number of fibre sections connecting the sensors broke. This was due to insufficient protection of the fibre from being crushed between two of the strands wires. This will be remedied in future work by enclosing the 
First-time demonstration of measuring concrete prestress levels with metal packaged distributed fibre optic sensors9

fibre in a plastic sleeve fixed into the strand groove. It is important to note that the sensors themselves survived the prestress levels applied to the strands and

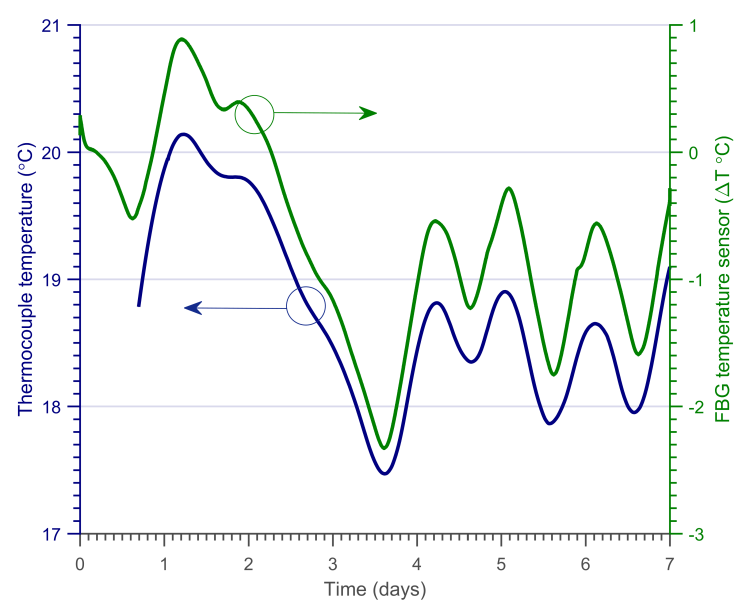

Figure 10. Temperature fluctuations monitored by the temperature FBG sensors and a thermocouple for the week after stressing the strands.
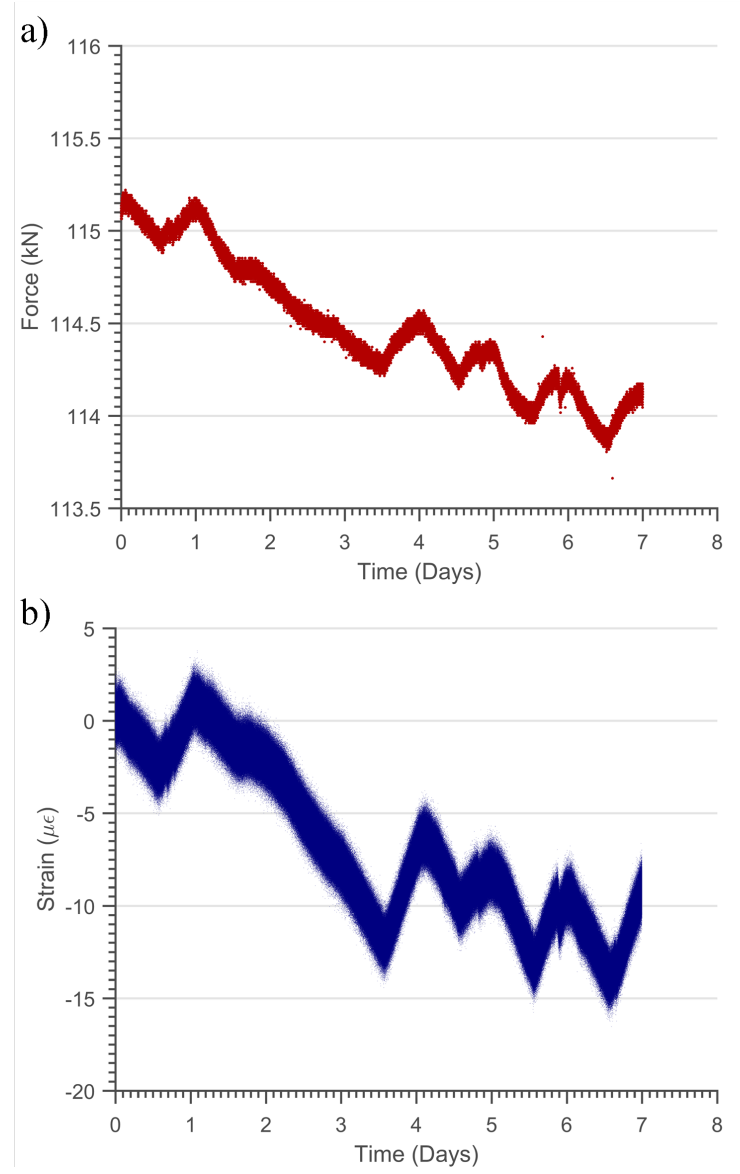

Figure 11. Example of load cell and strain sensor measuring the prestress loss in the week after stressing the strands (graph a) and b) respectively). the force applied to the strands was limited to $200 \mathrm{kN}$ because of the sensor line breakages. It has been shown previously that the sensors can survive while being attached to single wires subject to $1300 \mathrm{MPa}$ equivalent to $300 \mathrm{kN}$ for the 7 -wire strands $[23,22]$. For this reason, frictional effects on the distributed force of the strands could not be investigated.

\subsection{Force distribution between individual wires}

During calibration of the strain sensors, it was noted that the strain of individual wires did not linearly correlate with the total force of the strand for the complete force range. During previous tests of strain sensors mounted to individual wires a linear correlation was found. There are two distinct differences between this experiment and the previous ones; the sensors were spliced together in series and a 7 wire strand was used instead of a single wire. It is believed that the movement of individual wires and therefore uneven force distribution is the cause of the nonlinear relationship between the total force and strain of individual wires.

It is not known whether the king wire (central wire) takes most of the force during the initial stages of stressing the strand or if there is an even distribution of load between the wires. The sensor responses would be consistent with a large increase in force through an individual wire compared to the overall force in the strand. An uneven force distribution between the individual wires that varies as the load is increased would result in a non-linear response of an individual sensor compared to the overall force. Differences in strain between individual wires were discussed by Kim et al. and results presented here may provide additional evidence of this [10]. Further investigations are needed to determine the distribution of force between the wires and whether this varies as the strand load is increased. Moreover, if this was indeed the reason for the behaviour seen in the figures above, additional sensors would likely be required to sample more wires for the determination of the overall strain in a specific location along the strand.

\subsection{Future work}

For this technology to be adopted in a working containment building, a practical installation method must be devised and tested. The prestressing strands are stored on large cylindrical reels; sensors and the interrogating fibre could be mounted onto the strand by winding it from one reel to another. Tests would have to be made to check whether the fibre and sensors are able to withstand the radius of curvature of the reel. The number of sensors per strand would need to be increases so that the distributed force of the 
First-time demonstration of measuring concrete prestress levels with metal packaged distributed fibre optic sensors10

strand can be monitored. Additional sensors would reduce the cost per sensor as the interrogation system is relatively expensive compared to the individual sensors. Before installation it would be prudent to carry out an accelerated lifetime test of the sensor package in the presence of water and grease, in similar conditions to the application.

The long term response of the sensors and the concrete beams (in particular the bent ducts) and how this compares with prestress loss theory will be dedicated to future work. We will also investigate the force distribution between the wires by mounting several sensors in the same position on different wires. The ruggedness of the sensor addressing lines needs to be improved to ensure the sensors can be interrogated reliably. Additionally, work will be dedicated towards developing a calibration technique. Calibration by thermal expansion and three point bending will be investigated.

It should be noted that while these sensors have been developed specifically for nuclear containment buildings, they are equally applicable to other large structures such as precast bridges and offshore rigs. Furthermore, the sensors could also be deployed on pre-tensioned structures (PCPV's and PCC's are generally post-tensioned structures). Adequate sensor protection during the concrete pour would have to be implemented.

\section{Conclusion}

This paper outlines the methods used to instrument prestressing strands with FBG strain and temperature sensors allowing real time monitoring of prestress loss in ambient conditions. All metal sensor packaging and attachment was adopted to enable the sensors to survive the stresses involved and to reduce the poor bonding often encountered with use of epoxy. The sensors survived installation and stressing to $(\sim 1000 \mathrm{MPa})$. However, some of the sensor interrogation lines did not, indication that additional measures are needed to protect the fibre. Comparison of the strain measured by the strain sensors revealed that possibly individual wires in the strand share the total force unequally. Nevertheless, initial results show the strain sensors can measure the prestress loss. Future work will concentrate on investigating these two areas. We have shown it is possible to retrofit prestressing strands with FBG strain and temperature sensors. Application of this technology on a working nuclear site could improve the understanding of prestress systems in nuclear containment buildings.

\section{References}

[1] Smith L M 1996 The Structural Engineer 74 210-211
[2] Anderson P 2005 Nuclear Engineering and Design 235 2323-2336

[3] Mihailov S J 2012 Sensors 12 1898-1918

[4] Perry M, Niewczas P and Johnston M 2012 IEEE Sensors Journal 12 3248-3257

[5] Majumder M, Gangopadhyay T K, Chakraborty A K, Dasgupta K and Bhattacharya D 2008 Sensors and Actuators A: Physical 147 150-164

[6] Lopez-Higuera J M, Rodriguez Cobo L, Quintela Incera A and Cobo A 2011 Journal of Lightwave Technology 29 587-608

[7] Abdel-Jaber H and Glisic B 2014 Smart Materials and Structures 23075004

[8] Zhou Z, He J, Chen G and Ou J 2009 Journal of Intelligent Material Systems and Structures 20 1901-1912

[9] Lan C, Zhou Z and Ou J 2014 Structural Control and Health Monitoring 21 317-330

[10] Kim S, Park Y, Park S, Cho K and Cho J R 2015 Sensors 15 1060-1070

[11] Chin J, Aouadi K and Nguyen T 1997 Journal of Composites Technology \& Research 19 205-213

[12] Zaman A, Gutub S A and Wafa M A 2013 Journal of Reinforced Plastics and Composites 32 1966-1988

[13] Nawy E G 2010 Prestressed concrete A fundamental approach 5th ed (New Jersey, USA: Prentice Hall)

[14] British Standards Institution 1997 Structural use of concrete. (London: BSI)

[15] Mosley W, Hulse R and Bungey J 2012 Reinforced Concrete Design - to Eurocode 2 7th ed (Macmillan Education $\mathrm{UK}$ )

[16] ACI Committee 318 and American Concrete Institute 2014 Building code requirements for structural concrete (ACI 318-14): an ACI standard : commentary on building code requirements for structural concrete (ACI 318R14), an ACI report

[17] Hurst M K 1998 Prestressed concrete design (London; New York: E \& FN Spon)

[18] Hill K O and Meltz G 1997 Journal of lightwave technology $151263-1276$

[19] Zhou Z and Ou J 2005 Development of FBG sensors for Structural Health Monitoring in civil infrastructures Sensing issues in civil structural health monitoring (Springer) pp 197-207

[20] Kurkjian C R, Gupta P K and Brow R K 2010 International Journal of Applied Glass Science $127-37$

[21] Perry M, Niewczas P, Johnston M, Cook K and Canning J 2013 IEEE Sensors Journal 13 816-823

[22] Fusiek G, Perry M, Niewczas P and Johnston M 2014 Deformation monitoring in prestessing tendons using fibre Bragg gratings encapsulated in metallic packages SPIE 9157, 23rd International Conference on Optical Fibre Sensors vol 9157A6

[23] Perry M, Yan Z, Sun Z, Zhang L, Niewczas P and Johnston M 2014 Nuclear Engineering and Design 268 35-40

[24] Mei Z and Morris J 1992 Journal of Electronic Materials 21 401-407

[25] Tan Q and Lee Y C 1996 Soldering technology for optoelectronic packaging Electronic Components and Technology Conference, 1996. Proceedings., 46th (IEEE) pp 26-36

[26] Beranek M W, Rassaian M, Tang C H, St John C L and Loebs V A 2001 Advanced Packaging, IEEE Transactions on $\mathbf{2 4} 576-585$

[27] Majda P and Skrodzewicz J 2009 International Journal of Adhesion and Adhesives 29 396-404

[28] Baker S R, Rourke H N, Baker V and Goodchild D 1997 Lightwave Technology, Journal of 15 1470-1477

[29] Mckeeman I, Niewczas P and Johnston M 2015 Identifying fabrication defects of metal packaged fibre Bragg grating sensors for smart pre-stressing strands Environmental, 
1

2

First-time demonstration of measuring concrete prestress levels with metal packaged distributed fibre optic sensors 11

Energy and Structural Monitoring Systems (EESMS), 2015 IEEE Workshop on (IEEE) pp 61-65

[30] Mckeeman I, Fusiek G, Perry M, Niewczas P and Johnston M 2015 In-situ temperature calibration procedure for temperature and strain fibre Bragg grating sensors for monitoring pre-stressing strands SPIE 9634, 24th International Conference on Optical Fibre Sensors vol 9634 pp $96345 \mathrm{I}-96345 \mathrm{I}-4$

[31] Jiang W C, Gong J M, Chen H and Tu S T 2008 Journal of Pressure Vessel Technology 130041203

[32] Li H N, Zhou G D, Ren L and Li D S 2009 Journal of engineering mechanics $1351343-1353$

[33] Wang H P and Zhou Z 2012 Pac. Sci. Rev 14 248-252 